DOI:

10.1038/nri1979

\section{URLs}

RIG-I

http://www.ncbi.nlm.nih.gov/ entrez/query.fcgi?db=gene\&c $\mathrm{md}=$ Retrieve\&dopt=full_ report\&list_uids $=23586$ MDA5

http://www.ncbi.nlm.nih.gov/ entrez/query.fcgi?db=gene\&c $\mathrm{md}=$ Retrieve\&dopt=full_ report\&list_uids $=64135$

\title{
VIRAL IMMUNITY
}

\section{RIG-I reveals its perfect partner}

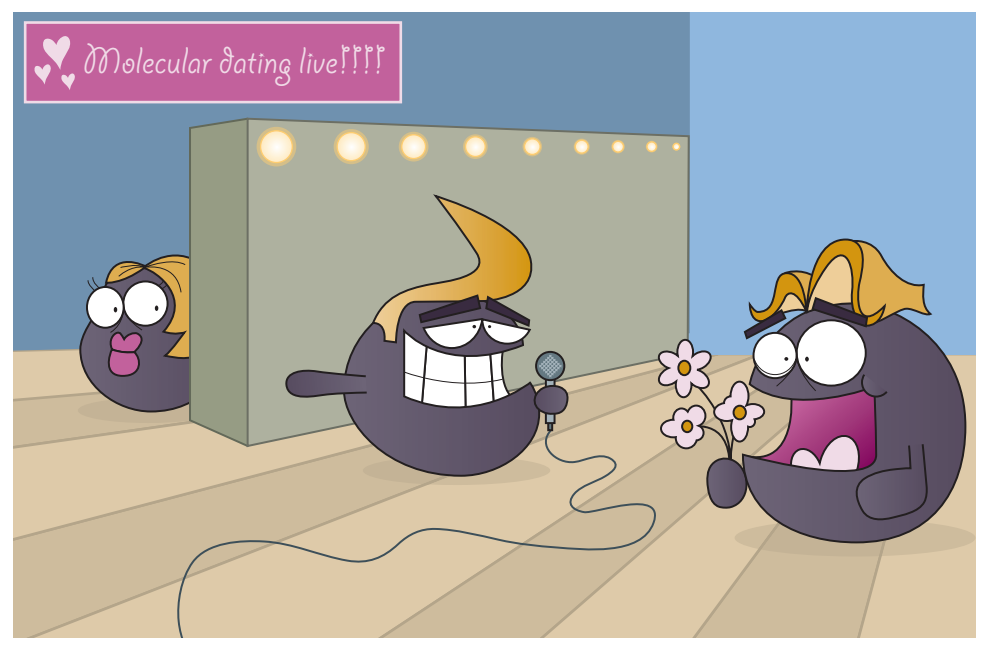

The potent type I interferon (IFN) response to viral infection has recently been shown to depend on the recognition of viral nucleic acids not only by membrane-associated Toll-like receptors, but also by the cytoplasmic RNA helicases RIG-I (retinoic-acid-inducible protein I) and MDA5 (melanoma differentiation-associated protein 5). However, it remains controversial how RIG-I and MDA5 discriminate between self and non-self RNAs. Two papers in Science report that, contrary to popular belief, $5^{\prime}$-triphosphate RNA, and not viral replication or the formation of double-stranded (ds)RNA, is the key to viral recognition by RIG-I.

Influenza A virus normally inhibits IFN production by infected cells through the action of the viral protein NS1. Pichlmair et al. showed that neither wild-type influenza A virus nor a mutant virus lacking NS1 produced dsRNA in infected cells. By contrast, NS1 was unable to inhibit the IFN response to Semliki Forest virus, which generates high levels of dsRNA.
Therefore, neither IFN induction nor the inhibitory effect of NS1 correlates with the presence of dsRNA, but IFN responses were induced by genomic single-stranded (ss)RNA purified from influenza virions (flu vRNA). Hornung et al. showed that in vitrotranscribed ssRNA and dsRNA with a $5^{\prime}$ triphosphate could induce IFN $\alpha$ production by monocytes, but that the response was abrogated by dephosphorylation of the $5^{\prime}$ end, synthetic 'capping' of the RNA or nucleoside modification of RNA. Similarly, in the study by Pichlmair et al., the IFN response to flu vRNA was abrogated by treatment with a phosphatase (presumably by dephosphorylation of the $5^{\prime}$ triphosphate). Therefore, both groups propose that the $5^{\prime}$ triphosphate in viral RNA might be responsible for IFN $\alpha$ induction by some viruses.

Hornung et al. showed that whereas wild-type and MDA5-deficient cells produce large amounts of IFN $\beta$ in response to $5^{\prime}$-triphosphate $\mathrm{RNA}$, no response was detected in
RIG-I-deficient cells or cells expressing a dominant-negative RIG-I mutant. By contrast, overexpression of RIG-I increased the IFN response. The role of RIG-I in the detection of 5'-triphosphate viral RNA was also shown by Pichlmair et al.; RIG-I directly bound flu vRNA in vitro but formed a less stable complex with phosphatase-treated vRNA. Also, both groups showed that a $5^{\prime}$-triphosphate RNA could immunoprecipitate RIG-I, but not if the $5^{\prime}$-triphosphate group was enzymatically removed.

Both groups conclude that non-self versus self discrimination of RNA by RIG-I depends on the presence or absence of a 5 '-triphosphate end, respectively. Many RNA viruses have uncapped RNAs bearing $5^{\prime}$ triphosphates, whereas uncapped, unmodified $5^{\prime}$ triphosphates are mainly absent from self RNAs owing to the addition of a 7-methyl-guanosine cap or nucleoside modification. However, picornaviruses, which are recognized by MDA5 rather than RIG-I, do not produce 5 '-triphosphate RNA. Notably, human 7SL RNA (which is present at high copy numbers in the cytosol but does not induce an IFN response) has a $5^{\prime}$-triphosphate end; this indicates that other features of RNA (such as nucleoside modification or unknown alternatives) must also contribute to the distinction of self and viral RNA by the RNA helicases.

Kirsty Minton

ORIGINAL RESEARCH PAPERS Pichlmair, A. et al. RIG-I-mediated antiviral responses to singlestranded RNA bearing 5' phosphates. Science 12 October 2006 (doi:10.1126/science.1132998) | Hornung, V.et al. 5'-triphosphate RNA is the ligand for RIG-I. Science 12 October 2006 (doi:10.1126/science.1132505) 